\title{
Costs associated with mood and anxiety disorders, as evaluated by telephone survey
}

\author{
SB Patten, MD, PhD (1); JVA Williams, MSc (1); C Mitton, MSc (2)
}

\begin{abstract}
Costing studies are central to health policy decisions. Available costing estimates for mood and anxiety disorders in Canada may, however, be out of date. In this study, we estimated a set of direct health care costs using data collected in a provincial telephone survey of mood and anxiety disorders in Alberta. The survey used random digit dialing to reach a sample of 3394 household residents aged 18 to 64. A telephone interview included items assessing costs without reference to whether these were incurred by the respondent, government or a health plan. The survey interview also included the Mini Neuropsychiatric Diagnostic Interview (MINI). Costs for antidepressant medications appear to have increased since the last available estimates were published. Surprisingly, most medication costs for antidepressants were incurred by respondents without an identified disorder. Also, an unexpectedly large proportion of medication costs were for psychotropic medications other than antidepressants and anxiolytic-sedative-hypnotics. These results suggest that major changes have occurred in the costs associated with antidepressant treatment. Available cost-of-illness data may be outdated, and some assumptions made by previous studies may now be invalid.
\end{abstract}

Key words: Cross-sectional studies, Depressive Disorders, Costs and Cost Analysis

\section{Background}

Depressive disorders present an important challenge to population health. ${ }^{1}$ According to the Global Burden of Disease Project, unipolar major depression is the $4^{\text {th }}$ leading contributor to disease burden world-wide, ranking second in developed countries such as Canada. ${ }^{2}$ The impact of this condition on population health relates partially to its high prevalence: approximately $5 \%$ of Canadians experience an episode of major depression in any given year. ${ }^{3}$ However, its impact is magnified by a peak prevalence in the 25-44 year age group $^{4}$ which is a critical period for education, establishment of relationships and economic productivity. Major depression is also a recurrent condition, and one that is frequently associated with psychiatric and medical comorbidity. ${ }^{5,6}$ All of these factors tend to magnify its impact on population health. Finally, major depression has an impact on mortality.?

Around the world, a variety of costing studies for depressive disorders have been carried out. Typically, the goal of these studies has been to estimate overall costs, including direct and indirect treatment costs. Population-based costing studies typically use data from a variety of sources such as government reports, administrative data and national surveys. This is because data collected from any particular source (e.g. a national survey) are unlikely to be comprehensive enough for detailed costing.

Recently, Thomas and Morris ${ }^{8}$ published cost-of-illness estimates for depression in England. Their study integrated data from a large number of sources and provides what has been considered the best available estimates for Europe. ${ }^{9}$ However, the approach taken to assess some costing elements was crude. For example, in the assessment of medication costs, treated prevalence was estimated using a formula that began with administrative data for physician visits. The estimated number of people making visits for depression was multiplied by the proportion of antidepressant medications prescribed nationally in specific antidepressant categories, and in turn, by the average costs of prescriptions in those categories. This approach assumes that the total cost can be conceptualized as the treated prevalence multiplied by the cost per treated person. The approach seems reasonable on the surface, but does not consider that antidepressant treatment is often continued long after the remission of depressive episodes. ${ }^{10}$

Stephens and Jouber ${ }^{11}$ estimated the total burden of major depression and distress in Canada in 1998, also using data from a variety of sources. Their overall cost estimate was $\$ 14.4$ billion. Their analysis was based partially on data from the National Population Health Survey (NPHS) and partially on data collected by the 1998 Economic Burden of Illness in Canada (EBIC) project (http://www.phac-aspc. gc.ca/publicat/ebic-femc98/index.html). All of the data on medication costs came from EBIC. In turn, the EBIC estimates were taken from a cost tracking database at the Canadian Institute for Health Information (CIHI) containing cost estimates from a private-sector company: IMS Health Canada. This approach is useful because IMS Health Canada databases can identify both the total volume of medication sales and the proportion of treatment recom-

\section{Author References}

1 University of Calgary, Department of Community Health Sciences, Calgary, Alberta

2 Health Studies, University of British Columbia, Kelowna, British Columbia

Correspondence: Scott B. Patten, University of Calgary, Department of Community Health Sciences, 3330 Hospital Drive NW, Calgary, Alberta T2N 4N1, Tel:

(403) 220-8752, Email: patten@ucalgary.ca 
mendations for specific indications. According to EBIC, \$108 million was spent on drugs for anxiety states, and \$252 million, for depressive disorders. However, this analysis is now 10 years old, and some of its estimates are nearly 15 years old. The ensuing period of time has seen increasing recognition of major depression as a health problem, likely diminishing stigma related to the condition and the emergence of new pharmacological and non-pharmacological treatments.

In 2003, sponsored by the Alberta Mental Health Board, a provincial mental health survey was conducted in Alberta, called the Alberta Mental Health Survey. ${ }^{12}$ In 2004 another initiative, sponsored by Alberta Health and Wellness and Administered by the Institute of Health Economics, called the Alberta Depression Initiative, was established. As a component of this latter initiative, a second provincial telephone survey focusing on mental health was carried out in the following year, this time with an emphasis on depression. Some of the survey results are relevant to future costing studies for mood and/or anxiety disorders in Canada and are summarized in this report.

\section{Methods}

\section{Survey Data Collection}

Alberta is a Canadian province with a population of 3.3 million residents, dispersed over an area of $661190 \mathrm{~km}^{2}$. Telephone survey methods were the most feasible strategy for obtaining an approximately representative sample in this geographically dispersed population. Approximately $2 / 3$ of the Alberta population resides in two cites: Edmonton and Calgary. The sampling procedure was stratified so that $1 / 3$ of the sample would come from each of these cities, with the balance coming from remaining rural areas. Data collection was restricted to household residents between the ages of 18 and 64 with a residential telephone line.

Data collection was carried out by the population survey unit of the Quality, Safety and Health Information (QSHI) portfolio in the Calgary Health Region (www.calgaryhealthregion.ca) during 2005. A listing of provincial, residential telephone numbers was initially sampled and the last digit was substituted with a random integer in order to increase coverage of unlisted numbers ${ }^{13,14}$ and to avoid bias that might be introduced if households with unlisted numbers differed from those with listed numbers. When a household was reached, a pseudo-random procedure, the "last birthday method" was used to randomly select a single subject from the household. As many as nine call-backs were made in an effort to reach all sampled individuals. These calls were distributed over working hours, evening and weekends.

The Mini Neuropsychiatric Interview (MINI), a brief structured diagnostic interview, ${ }^{15,16}$ was used as a diagnostic indicator for Major Depression (MD) and a set of other common mood and anxiety disorders. For major depressive episodes, past-14-day prevalence was assessed (essentially, the point prevalence for this disorder), for dysthymia, past-2-year prevalence was assessed. For panic disorder, agoraphobia, generalized anxiety disorder and social phobia, current prevalence was also assessed. Since it has been shown that differences between survey instruments often relate to the role of "clinical significance" probes in the scoring algorithms, ${ }^{17}$ we incorporated an interference item asking respondents whether their psychiatric symptoms interfered with their life. Episodes were considered clinically significant if respondents reported “a lot” of interference.

A pharmacoepidemiology module and a module designed to identify non-pharmacological treatment were also included in the interview. The pharmacoepidemiology module operated with a cyclical item-flow structure, initially asking about medications taken for the treatment of broadly defined but relevant symptoms ("Do you currently take any prescription medications for anxiety, depression, stress, energy levels, sleeping, pain management, fibromyalgia or migraine headaches?"), and then looping through each reported medication with a series of items inquiring about the number and size of tablets, reasons for use of the medication and duration of use. Respondents were prompted to report information directly from their pill bottle labels to ensure accuracy of this information. Dosage was determined by combining information about the size(s) and number of relevant tablets or capsules taken, including pro re nata (prn) schedules. The dosages were recorded in milligrams per day. The survey interview also included items evaluating the frequency of use of health services. These items were typically paired, with an initial item asking about use of the service and a second quantifying the frequency of use. For example, one item asked: "In the past 6 months have you consulted with a specialist physician other than a general practitioner, family doctor, emergency room physician or psychiatrist about your health?" If the answer was "yes" this was followed by "How many times?" Similar items assessed visits with family physicians, psychiatrists, psychologists and social workers, as well as emergency and walk-in clinic attendance, radiological procedures, and hospitalizations.

\section{Costing Procedures}

We obtained costs per milligram for each medication from the provincial drug plan costing guide (http://www.pao.gov.ab.ca/ benefits/choice/prescription-drug-plan. pdf). In situations where various dosage forms had different per milligram costs, we calculated the cost for the lowest milligram dosage for each drug. The costs associated with generic preparations were used when a generic preparation was available, since pharmacists can substitute the lowest cost alternative when filling a prescription. When a person was taking more than one drug from a particular class, the costs were estimated in the same way for each drug, and the costs of individual drugs were added together. For ease of interpretation, the daily costs were multiplied by 365 to project an annual medication cost. This approach seemed reasonable since $80 \%$ of survey respondents who reported taking antidepressants in the survey reported taking them for longer than one year. The same procedure was followed for anxiolytic-sedative-hypnotic and antipsychotic medications. 
For utilization of professional services, fee codes for the various professional groups were used. For physicians, fee codes from the Alberta Health Care Insurance Plan (AHCIP) were used. For family physicians, a basic code for an office visit not requiring a complete history and evaluation was used (fee code 03.03A). Emergency room visits were handled separately, using an estimated cost per single visit at twice the fee for a visit to a family physician. For specialist physicians, the fee code for a single referred consultation visit for 15 minutes was used (fee code 03.03FA). Fees associated with 45 minutes of clinical management time were used for psychiatrists (skill level code 08.19A) and for psychologists, fee schedules published by the Provincial Psychological Association were used.

It was not feasible to itemize radiological procedures and laboratory tests reported by survey participants. Instead, representative mid-point costing estimates were identified for common or typical procedures within each category. In this study these were: a chest X-ray, basic CT of the head and a basic MRI of the head. The per procedure costing estimates used in the study came from a costing guide produced by the Calgary Health Region. This guide is intended to ensure cost recovery in research projects, including materials and supplies and personnel-related costs. Similarly, mean per diem costs associated with hospitalizations from across the province used estimates developed by the Calgary Health Region for administrative purposes. The documents from the Calgary Health Region were considered to be a reasonable source since the Region includes the city of Calgary, but also rural areas stretching southwest of the city to the border with British Columbia.

For each category, a cost projection to the total population was made. The weighted prevalence for the mood and/or anxiety disorder diagnostic category was multiplied by the total provincial population in the studied age range, the proportion using the specific procedure, treatment or service and by the mean cost associated with it to produce a final cost estimate. Sampling weights were calculated as the inverse of an estimated selection probability within age and sex categories in each of the three sampling regions for the provincial population. The resulting weight was further adjusted by multiplying it by the ratio of the number of eligible residents in each household divided by the number of telephone lines going into the household. The weights were treated as relative weights in the analysis.

\section{Results}

In total, 18113 telephone numbers were called. More than half of these were disqualified from the sample for the following reasons: 1663 households had no eligible residents, 329 were blocked calls, 846 reached only answering machines or voice mailboxes (i.e. no person could be contacted with call-backs), 1747 were business lines, 3333 were not in service, 1385 reached fax machines, 378 reached non-English-speaking households, 845 were never answered during the call-back protocols, 11 met with hostile interruptions and 79 were disqualified for miscellaneous reasons. There were 7497 calls that successfully reached eligible households. At the household level, there were 3443 refusals $(45.9 \%)$. Of the 4054 households from which respondents could be selected, there were 635 individual refusals (15.7\%). Of the 3419 consenting respondents, interviews were completed in all but $25(0.7 \%)$, so that 3394 interviews were completed. After checks for data completeness and accuracy, 45 records were removed from the data set because of concerns about data quality. The final analysis included data collected from the remaining 3345 individuals $(82.5 \%$ of individuals who were invited to participate and provided adequate data). If the response rate is calculated using the number of eligible households in the denominator, however, it is $44.6 \%$. Using the Marketing Research and Intelligence Association (MRIA) method of calculation (http://www.tpsgc-pwgsc. gc.ca/por/text/pebptel-intro-e.html), 7628 of the 18113 can be classified as "out of scope" leading to a response rate of $31.9 \%$.

There were 168 survey participants with a mood disorder (current major depression or dysthymia) according to the MINI, leading to a prevalence estimate of $4.6 \%$. This estimate is consistent with existing literature. The 30-day prevalence of major depression in the Canadian Community Health Survey, Mental Health and Wellbeing (CCHS 1.2) was $1.8 \% .^{4}$ The CCHS 1.2 did not assess dysthymic disorder, but a systematic review by Waraich estimated a past-year prevalence of approximately $2 \% .{ }^{18}$ One hundred and ninety two respondents had one or more anxiety disorders, leading to a prevalence estimate of $6.3 \%$. This is slightly higher than the CCHS 1.2 past-year estimate of anxiety disorder prevalence (4.7\%), ${ }^{19}$ but the CCHS 1.2 did not assess generalized anxiety disorder, which has an annual population prevalence of $3.1 \%$ in the US. ${ }^{20}$ Most respondents with disorders had comorbid mood and anxiety disorders. Only $2.2 \%$ had a mood or anxiety disorder without having both. $4.1 \%$ had comorbid mood and anxiety disorders. Since the MINI is a brief screening instrument, its ability to distinguish between mood and anxiety disorders is questionable. The analysis therefore focussed on two groups: respondents with a mood or anxiety disorder (but not both) and respondents with a comorbid mood and anxiety disorder.

With respect to medication use at the time of the survey, $7.4 \%$ of the population reported taking an antidepressant, 3.1\% reported taking a sedative hypnotic medication and $1.5 \%$ took another medication potentially related to the management of mood disorders: mood stabilizers, psychostimulants or atypical antipsychotic medications. Although the latter group of medications are pharmacologically distinct, they were grouped together in the subsequent analysis because there were insufficient numbers to examine these categories separately. It should also be noted that the question stems indicated that the medications of interest were those taken for psychotropic purposes, and this category 
would not include, for example, anticonvulsant mood stabilizers taken for the treatment of epilepsy.

Of the $2.2 \%$ of the population with noncomorbid mood or anxiety disorders, $20.4 \%$ were taking one or more antidepressant medications. Of those with comorbid mood and anxiety disorders, $44.4 \%$ were taking one or more antidepressant medications. In the group with no detected mood or anxiety disorders, 5.6\% were taking an antidepressant. The estimated mean annual cost for antidepressants in each of these groups is summarized in Table 1 . At the time of the survey, there were 2105167 provincial residents in the 18 to 64 age range. The average cost per person multiplied by the estimated prevalence and number of people in the province in each diagnostic category led to a total cost estimate of $\$ 124$ million for antidepressants. This included \$7 million for people with a noncomorbid mood or anxiety disorder, \$35 million for people with comorbid disorders and $\$ 83$ million for those with no MINI-detected disorder.

Although the frequency with which antidepressants were taken by respondents without detected mood or anxiety disorders was relatively low at $5.6 \%$, and while the projected annual per person cost in this group was slightly lower than the group with MINI-detected disorders (see Table $1)$, the overall cost in this group was highest because the number of people in this category was the highest. This suggests that most of the costs associated with antidepressant treatment now occur in the continuation and maintenance phase ${ }^{10}$ of treatment since these respondents no longer met symptomatic criteria for mood or anxiety disorders. The result is in itself not necessarily surprising since acute treatment with antidepressants usually takes 6 to 8 weeks, whereas maintenance treatment lasting one year or longer is generally recommended, and indefinite treatment may be indicated in those with highly recurrent disorders. ${ }^{10}$ There are however, other possible explanations for this result, as discussed below.

A similar pattern was seen for sedativehypnotic medications (see Table 1). In people with mood or anxiety disorders, the frequency of use of these medications was much higher than that of respondents without these disorders. However, most people taking these medications did not have a mood or anxiety disorder. Hence, most of the costs occurred in the no-disorder group. The estimated total cost of sedativehypnotic use in the population was $\$ 8$ million, much lower than the cost of antidepressants.

A variety of other medications were reported by the survey respondents: lithium ( $\mathrm{n}=7)$, carbamazepine ( $n=3)$, valproate $(n=5)$, gabapentin ( $\mathrm{n}=9)$, lamotrigine $(\mathrm{n}=1)$, topiramide $(\mathrm{n}=20)$, dexamphetamine ( $n=6)$, chlorpromazine ( $n=1)$, olanzapine $(n=10)$, quetiapine $(n=8)$, clozapine $(n=2)$ and risperidone $(n=20)$. Ninetytwo respondents reported taking one or more of these medications. Unfortunately, only 69 of these respondents (75\%) were willing or able to provide dosage information. Considered as a group, an estimated $1.5 \%$ of the total population was taking one or more medications from this category. The frequency of use in respondents with non-comorbid mood or anxiety disorders was similar to that of the total population (see Table 1), but the frequency was much higher in the group with comorbid disorders $(16.4 \%)$. Approximately $1 \%$ of the population without a mood or anxiety disorder was taking one of these medications approximately consistent with the prevalence of psychotic disorders in the population. The projected total cost was \$38 million, \$25 million of which was accounted for by the group with no detected disorder. These results suggest, however, that a sizable proportion of treatment costs for mood and anxiety disorders may now occur outside of the pharmaceutical classes traditionally believed to be most important in the treatment of mood and anxiety disorders: antidepressant medications and sedative-hypnotic medications.

Table 2 presents the costs associated with utilization of services provided by mental health professionals. The frequency with which the services of psychiatrists and psychologists were utilized was much higher in people with mood and anxiety disorders, especially in the comorbid category. Having a mood or anxiety disorder was associated with a three-fold increase in these costs, whereas a more than eight-fold increase was seen in the comorbid group. However, most of the costs for psychologists occurred in the group with no MINI-defined disorder, suggesting that these professionals may often be engaged in treatment activities not specifically directed at these disorders, e.g. marital therapy. Table 3 presents the costs associated with service utilization for family physicians and specialist physicians other than psychiatrists. The respondents with mood and anxiety disorders were more likely to see a physician, and to have more visits. Both factors contributed to higher per-respondent costs in the group with mood and anxiety disorders.

Costing data for diagnostic tests are presented in Table 4 . In the case of radiological procedures, the frequency of testing (and hence, per-subject costs according to the procedures employed in this study) did not differ by disorder category. However, the proportion of subjects in the various subgroups having these tests was higher in subjects with mood and anxiety disorders. As shown in Table 5, the costs associated with emergency room visits and hospitalizations were also elevated in respondents having comorbid mood and anxiety disorders.

\section{Conclusions}

Many of the results presented here are consistent with an existing literature of costing studies for depressive disorders. They illustrate that costs associated with medication use and utilization of services are increased in people with these disorders. As expected, this was true both for measures of mental health care and for general medical care. An important interpretive issue is that mood disorders are strongly associated with long-term medical conditions, ${ }^{21-23}$ such that these increased costs may reflect an indirect effect of nonpsychiatric conditions. When one considers that approximately $10 \%$ of the Canadian population live in Alberta, the results suggest a substantial increase in the costs of pharmacotherapy for depression since the publication of the 1998 EBIC. In the 
EBIC, the total national costs for medications for mood and anxiety disorders was only twice as high as that found in the province of Alberta alone. In other words, if one assumes that $10 \%$ of the $\$ 360$ million estimated by the EBIC was spent in Alberta, the provincial estimate would be approximately $\$ 36$ million; however, the current study indicates that the cost of antidepressant medications in Alberta may be between 3 and 4 times higher than that. The increase in cost is not unexpected, as both the number of retail prescriptions for medications and the average cost per prescription are increasing in Canada. ${ }^{24}$

Although antidepressants and sedativehypnotic medications were the most frequently taken medications, an appreciable number of respondents reported taking other medications such as atypical antipsychotics. These medications now appear to make a substantial contribution to total treatment costs. Future analyses should include costs for atypical antipsychotics, stimulants and mood stabilizers.

Another interesting finding is the high proportion of costs in the no-disorder group. It should be emphasized that the MINI would not detect treated disorders that are in remission, so in some proportion of instances these costs may represent appropriate use of the medications. However, it is likely that some of the medication use in the no-disorder group is inappropriate (i.e. no disorder indicating treatment is present). Symptoms of depression and anxiety can occur in response to losses, threats and stressors. The Canadian health system is not well-structured for delivering the brief psychological interventions that would generally be appropriate in such cases. In Canada, primary mental health care is usually funded on a fee-for-service model. Primary care practices in Canada typically see large volumes of patients for brief visits. Antidepressants may sometimes be prescribed inappropriately due to a lack of time or expertise on the part of primary care physicians. Similarly, the use of sedative-hypnotic and atypical antipsychotic medications in the comorbid group may partially reflect poor access to non-pharmacologic treatments such as sleep hygiene and relaxation techniques. Finally, some proportion of medication use in this group may be due to treatment for conditions other than mood and anxiety disorders. For example, antidepressants are frequently used for chronic pain and migraine prophylaxis.

The results may have important implications for future costing studies. For example, the UK study discussed above ${ }^{8}$ used billing data to identify treated prevalence, but people on long-term maintenance treatment may not have visits coded specifically for a mood or anxiety disorder. In fact, any approach that begins with prevalence data is likely to miss a large proportion of medication costs, since the treated disorders may be in remission and may also be missed in symptom-based prevalence measures used in surveys.

There are several limitations associated with this study. First, the study focused on psychotropic medication use. Since some chronic medical conditions are associated with mood and anxiety disorders, it is likely that increased costs associated with non-psychiatric procedures and treatments would occur in the subjects with mood and anxiety disorders. It was not considered feasible to record all medications being

TABLE 1

Annual and six-month costs associated with medication use, by disorder category

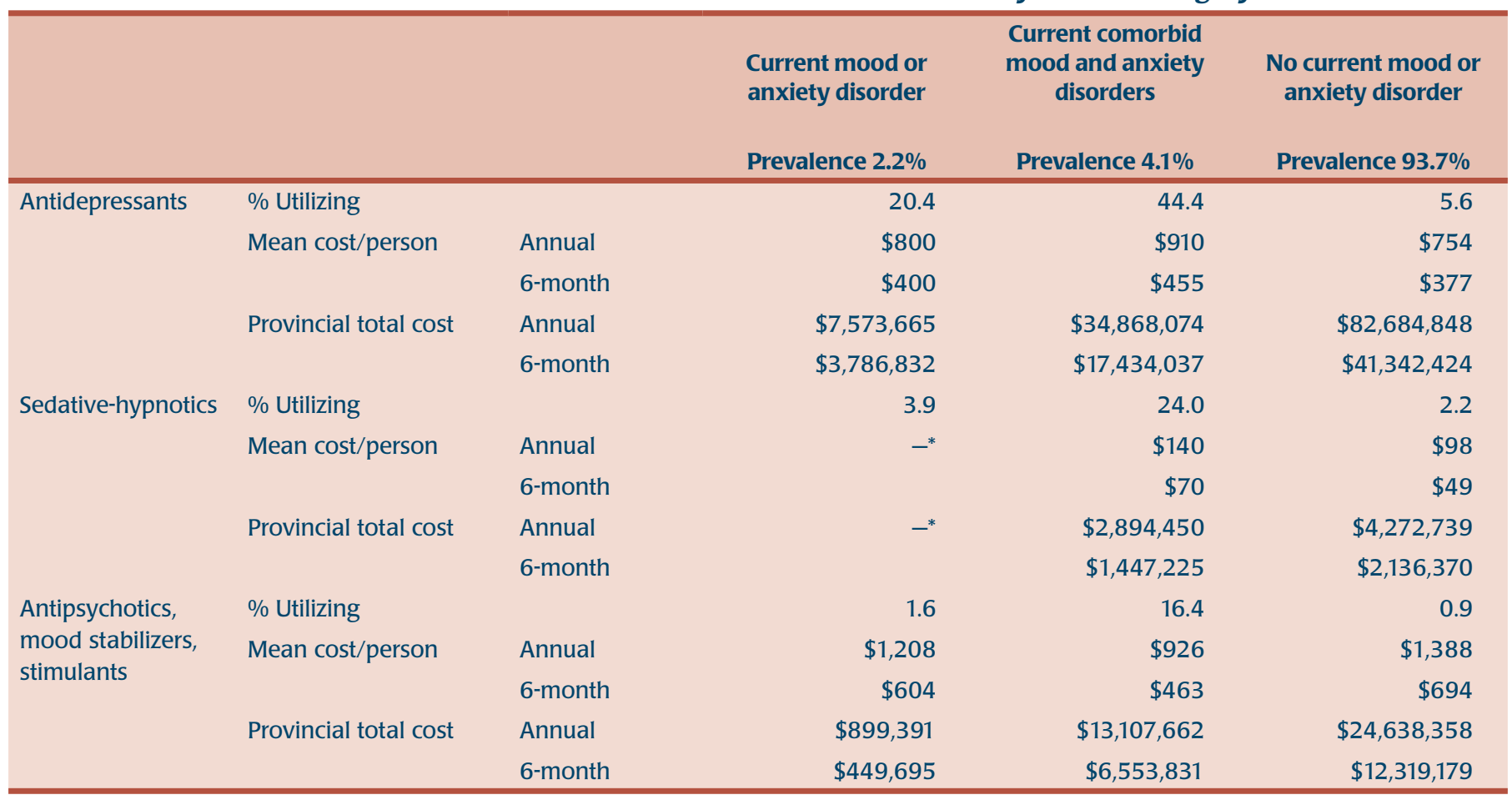

${ }^{*}$ numbers too small to support estimation. 
TABLE 2

Utilization of services provided by mental health specialists, by disorder category (during the 6 months prior to the survey)

\begin{tabular}{|c|c|c|c|c|}
\hline & & $\begin{array}{c}\text { Current mood } \\
\text { or anxiety } \\
\text { disorder } \\
\text { Prevalence } \\
2.2 \%\end{array}$ & $\begin{array}{c}\text { Current } \\
\text { comorbid } \\
\text { mood and } \\
\text { anxiety } \\
\text { disorders } \\
\text { Prevalence } \\
4.1 \%\end{array}$ & $\begin{array}{c}\text { No current } \\
\text { mood or } \\
\text { anxiety } \\
\text { disorder } \\
\text { Prevalence } \\
93.7 \%\end{array}$ \\
\hline \multirow[t]{4}{*}{ Psychiatrist } & \% Utilizing & 6.1 & 26.1 & 1.8 \\
\hline & Mean number of visits & 2.4 & 10.4 & 3.2 \\
\hline & Mean cost/person & $\$ 351$ & $\$ 1,501$ & $\$ 467$ \\
\hline & Provincial total cost & $\$ 996,754$ & $\$ 33,811,137$ & $\$ 16,544,730$ \\
\hline \multirow[t]{4}{*}{ Psychologist } & $\%$ Utilizing & 6.2 & 14.5 & 1.7 \\
\hline & Mean number of visits & 4.7 & 6.6 & 4.2 \\
\hline & Mean cost/person & $\$ 698$ & $\$ 987$ & $\$ 632$ \\
\hline & Provincial total cost & $\$ 2,013,150$ & $\$ 12,354,147$ & $\$ 21,110,090$ \\
\hline
\end{tabular}

taken by the subjects during the telephone interview, however. Second, the recording of costs was certainly not exhaustive. In particular, indirect costs were not assessed. Whereas various categories of cost were estimated in respondents with and without evidence of a disorder, it does not follow that the disorders are necessarily the cause for the costs. For example, we looked at the costs associated with hospitalization for any reason. Since chronic illnesses are associated with mood disorders, the hospital costs may more closely reflect these medical comorbidities than the depression itself. Mechanisms linking depression to health care costs are likely to be complex. For example, a negative effect of depression on self-management of chronic conditions could lead to an increased frequency of hospital admissions. It is doubtful whether such costs can confidently be attributed to a specific diagnostic category.

Additional limitations pertain to the use of telephone survey methods. Declining response rates to telephone surveys in recent decades have heightened concerns about vulnerability of such studies to bias (see recent report by Public Works and Government Services Canada at http:// www.pwgsc.gc.ca/por/text/pebptel-introe.html). Because brevity is needed in telephone survey interviews, the assessment of utilization was necessarily crude. However, telephone-based data collection did allow the collection of data from a large sample dispersed across a large geographical region, and this included more detailed information about medication use than has generally been available in prior costing studies. However, response rates were less than ideal, raising questions about the validity of the estimates. Also, the interviewers encountered some difficulty with the collection of detailed information about dosages from respondents who were taking multiple medications or medications that involve variable dosages or prn schedules. A high frequency of missing data, for example, in the case of medications

TABLE 3

\section{Costs associated with service utilization, family and specialist physicians (not including psychiatrists), by disorder category (during the $\mathbf{6}$ months prior to the survey)}

\begin{tabular}{|c|c|c|c|c|}
\hline & & $\begin{array}{l}\text { Current mood } \\
\text { or anxiety } \\
\text { disorder } \\
\text { Prevalence } \\
2.2 \%\end{array}$ & $\begin{array}{c}\text { Current } \\
\text { comorbid } \\
\text { mood and } \\
\text { anxiety } \\
\text { disorders } \\
\text { Prevalence } \\
4.1 \%\end{array}$ & $\begin{array}{c}\text { No current } \\
\text { mood or } \\
\text { anxiety } \\
\text { disorder } \\
\text { Prevalence } \\
93.7 \%\end{array}$ \\
\hline \multirow{4}{*}{$\begin{array}{l}\text { Family } \\
\text { physician }\end{array}$} & $\%$ Utilizing & 58.1 & 74.5 & 53.1 \\
\hline & Mean number of visits & 4.9 & 7.2 & 2.8 \\
\hline & Mean cost/person & $\$ 113$ & $\$ 168$ & $\$ 130$ \\
\hline & Provincial total cost & $\$ 3,076,347$ & $\$ 10,815,008$ & $\$ 68,375,547$ \\
\hline \multirow{4}{*}{$\begin{array}{l}\text { Other } \\
\text { physician }\end{array}$} & $\%$ Utilizing & 28.7 & 42.8 & 22.0 \\
\hline & Mean number of visits & 2.2 & 5.8 & 2.8 \\
\hline & Mean cost/person & $\$ 80$ & $\$ 216$ & $\$ 105$ \\
\hline & Provincial total cost & $\$ 3,725,192$ & $\$ 18,653,716$ & $\$ 208,201,753$ \\
\hline
\end{tabular}


TABLE 4 Diagnostic testing, by disorder category (during the 6 months prior to the survey)

\begin{tabular}{|c|c|c|c|c|}
\hline & & $\begin{array}{c}\text { Current mood } \\
\text { or anxiety } \\
\text { disorder } \\
\text { Prevalence } \\
2.2 \%\end{array}$ & $\begin{array}{c}\text { Current } \\
\text { comorbid } \\
\text { mood and } \\
\text { anxiety } \\
\text { disorders } \\
\text { Prevalence } \\
4.1 \%\end{array}$ & $\begin{array}{c}\text { No current } \\
\text { mood or } \\
\text { anxiety } \\
\text { disorder } \\
\text { Prevalence } \\
93.7 \%\end{array}$ \\
\hline \multirow[t]{4}{*}{ X-ray } & \% Utilizing & 17.6 & 28.9 & 14.4 \\
\hline & Mean number of tests & 1.6 & 2.6 & 2.3 \\
\hline & Mean cost/person & $\$ 142$ & $\$ 226$ & $\$ 165$ \\
\hline & Total cost & $\$ 1,164,288$ & $\$ 5,636,873$ & $\$ 46,827,952$ \\
\hline \multirow[t]{4}{*}{ CT scan } & \% Utilizing & 2.8 & 11.6 & 2.6 \\
\hline & Mean number of tests & 1.3 & 1.5 & 1.2 \\
\hline & Mean cost/person & $\$ 224$ & $\$ 255$ & $\$ 209$ \\
\hline & Total cost & $\$ 292,946$ & $\$ 2,551,402$ & $\$ 10,669,647$ \\
\hline \multirow[t]{4}{*}{ MRI } & $\%$ Utilizing & 1.0 & 10.7 & 3.2 \\
\hline & Mean number of tests & 1.9 & 1.2 & 1.1 \\
\hline & Mean cost/person & $\$ 1,206$ & $\$ 749$ & $\$ 669$ \\
\hline & Total cost & $\$ 561,240$ & $\$ 6,915,813$ & $\$ 42,095,483$ \\
\hline \multirow[t]{4}{*}{ Lab tests } & \% Utilizing & 38.8 & 55.4 & 33.7 \\
\hline & Mean number of tests & 2.5 & 7.8 & 2.3 \\
\hline & Mean cost/person & $\$ 22$ & $\$ 70$ & $\$ 20$ \\
\hline & Total cost & $\$ 401,824$ & $\$ 3,329,481$ & $\$ 13,278,868$ \\
\hline
\end{tabular}

and anxiety disorders are less expensive than hospitalizations for other reasons, bias could be introduced.

In Canada, future costing studies will need to maximize the use of available data about the frequency of maintenance treatment and adopt a broad view of drug costs in order to obtain valid cost estimates. The economic "landscape" associated with mood and anxiety disorders appears to be evolving over time and future costing studies will need to accommodate these new realities.

\section{Acknowledgements}

This project was funded by the Institute of Health Economics through the Alberta Depression Initiative. Dr. Patten is supported by a Health Scholar award from the Alberta Heritage Foundation for Medical Research and is a Fellow with the Institute of Health Economics. Dr. Mitton is supported by the Michael Smith Foundation for Health Research and holds a Canada Research Chair in Health Care Priority Setting.

TABLE 5

Emergency room visits and hospitalizations, by disorder status (during the 6 month prior to the survey)

\begin{tabular}{|c|c|c|c|c|}
\hline & & $\begin{array}{l}\text { Current mood or } \\
\text { anxiety disorder }\end{array}$ & $\begin{array}{l}\text { Current comorbid mood } \\
\text { and anxiety disorders }\end{array}$ & $\begin{array}{l}\text { No current mood or } \\
\text { anxiety disorder }\end{array}$ \\
\hline & & Prevalence $2.2 \%$ & Prevalence $4.1 \%$ & Prevalence $93.7 \%$ \\
\hline \multirow[t]{3}{*}{ Emergency room visits } & $\%$ with a visit & 12.3 & 26.9 & 10.7 \\
\hline & Mean number of visits & 1.3 & 3.1 & 1.5 \\
\hline & Mean cost/person & $\$ 61$ & $\$ 144$ & $\$ 68$ \\
\hline \multirow[t]{4}{*}{ Hospitalizations } & $\%$ with a hospitalization & 9.4 & 23.7 & 5.6 \\
\hline & Mean number of hospital days & 4.7 & 23.5 & 5.0 \\
\hline & Mean cost/person & $\$ 4,988$ & $\$ 25,151$ & $\$ 5,338$ \\
\hline & Total cost & $\$ 21,714,793$ & $\$ 514,496,354$ & $\$ 587,712,652$ \\
\hline
\end{tabular}




\section{References}

1. Insel TR, Fenton WS. Psychiatric epidemiology. It's not just about counting anymore. Arch Gen Psychiatry. 2005;62:590-3.

2. Ayuso-Mateos JL. Global burden of unipolar depressive disorders in the year 2000. Global Burden of Disease Draft 28-05-03. World Health Organization Global Program on Evidence for Health Policy. 2003.

3. Statistics Canada. Canadian Community Health Survey Mental Health and Well-being. Ottawa: Statistics Canada, 2004 Mar 9.

4. Patten SB, Wang JL, Williams JVA, Currie SR, Beck CA, Maxwell CJ, et al. Descriptive epidemiology of major depression in Canada. Can J Psychiatry. 2006;51:84-90.

5. Blazer DG, Kessler RC, McGonagle KA, Swartz MS. The prevalence and distribution of Major Depression in a national community sample: the National Comorbidity Survey. Am J Psychiatry. 1994;151:979-86.

6. Kessler RC, Berglund P, Demler O, Jin R, Koretz D, Merikangas KR, et al. The epidemiology of major depressive disorder: results from the National Comorbidity Survey Replication (NCS-R). JAMA. 2003; 289:3095-105.

7. Wulsin LR, Vaillant GE, Wells VE. A systematic review of the mortality of depression. Psychosom Med. 1999;61:6-17.

8. Thomas CM, Morris S. Cost of depression among adults in England in 2000. Br J Psychiatry. 2003;183:514-9.

9. Andlin-Sobocki P, Wittchen H-U. Cost of affective disorders in Europe. Eur J Neurol. 2005;12(Suppl. 1):34-8.
10. Kennedy SH, Lam RW, Cohen NL, Ravindran $\mathrm{AV}$, and the CANMAT Depression Work Group. Clinical guidelines for the treatment of depressive disorders. IV. Medications and other biological treatments. Can J Psychiatry. 2001;46(Suppl 1):38S-58S.

11. Stephens T, Dulberg C, Joubert N. Mental health of the Canadian population: a comprehensive analysis. Chronic Dis Can. 1999;20:118-26.

12. Patten SB, Adair CE, Williams JVA, Brant $\mathrm{R}$, Wang JL, Casebeer A, et al. Assessment of mental health and illness by telephone survey: Experience with an Alberta Mental Health Survey. Chronic Dis Can. 2006; 27(3):99-109

13. Mullet GM. The efficacy of plus-one dialing: Self reported status. American Statistical Association: Proceedings of the Section on Survey Research Methods 1982;575-6.

14. Potthoff RF. Telephone sampling in epidemiological research: to reap the benefits, avoid the pitfalls. Am J Epidemiol. 1994; 139:967-78.

15. Lecubier Y, Sheehan DV, Weiller E, Amorim $\mathrm{P}$, Bonora I, Sheehan $\mathrm{KH}$, et al. The MINI International Neuropsychiatric Interview (MINI). A short diagnostic structured interview: reliability and validity according to the CIDI. Eur Psychiatry. 1997;12:224-31.

16. Sheehan DV, Lecubier Y, Sheehan KH, Janavs J, Weiller E, Keskiner A, et al. The validity of the Mini International Neuropsychiatric Interview (MINI) according to the SCID-P and its validity. Eur Psychiatry. 1997;12:232-41.

17. Narrow WE, Rae DS, Robins LN, Regier DA. Revised prevalence estimates of mental disorders in the United States: using a clinical significance criterion to reconcile 2 surveys' estimates. Arch Gen Psychiatry. 2002;59:115-23.
18. Waraich PS, Goldner EM, Somers JM, Hsu L. Prevalence and incidence studies of mood disorders: a systematic review of the literature. Can J Psychiatry. 2004;49:124-38.

19. Kessler RC, Chiu WT, Demler O, Merikangas KR, Walters EE. Prevalence, severity, and comorbidity of 12-month DSM-IV disorders in the National Comorbidity Survey Replication. Arch Gen Psychiatry. 2005 Jun; 62(6):617-27.

20. Statistics Canada. Canadian Community Health Survey: Mental health and wellbeing. The Daily, Wednesday, September 3, 2003. URL: http://www.statcan.ca/ Daily/English/030903/d030903a.htm

21. Patten SB, Beck CA, Kassam A, Williams JVA, Barbui C, Metz LM. Long-term medical conditions and major depression: strength of association in the general population. Can J Psychiatry. 2005;50:195-202.

22. Gagnon LM, Patten SB. Major depression and its association with long-term medical conditions. Can J Psychiatry. 2002;47:167-73.

23. Wells KB, Golding JM, Burnam MA Psychiatric disorder in a sample of the general population with and without chronic medical conditions. Am J Psychiatry. 1988;145(8):976-81.

24. Pharmaceutical trends: Top products, classes and companies sourced from IMS data. URL: http://www.imshealthcanada. com/web/channel/0,3147,77303623_6387 2702_77770072,00.html 